Article

\title{
Investigating the Thermodynamic Performances of TO-Based Metamaterial Tunable Cells with an Entropy Generation Approach
}

\author{
Guoqiang $\mathrm{Xu}^{1}{ }^{1}$, Haochun Zhang ${ }^{1, *}$, Xiu Zhang ${ }^{1}$ and Yan Jin ${ }^{2}$ \\ 1 School of Enengy Science and Engineering, Harbin Institute of Technology, Harbin 150001, China; \\ 16B902053@stu.hit.edu.cn (G.X.); zhangxiu_hit@163.com (X.Z.) \\ 2 Institute of Thermo-Fluid Dynamics, Hamburg University of Technology, Hamburg D-21073, Germany; \\ Jinyan995072@gmail.com \\ * Correspondence: zhc5@vip.163.com; Tel.: +86-451-8641-2328
}

Received: 26 August 2017; Accepted: 9 October 2017; Published: 13 October 2017

\begin{abstract}
Active control of heat flux can be realized with transformation optics (TO) thermal metamaterials. Recently, a new class of metamaterial tunable cells has been proposed, aiming to significantly reduce the difficulty of fabrication and to flexibly switch functions by employing several cells assembled on related positions following the TO design. However, owing to the integration and rotation of materials in tunable cells, they might lead to extra thermal losses as compared with the previous continuum design. This paper focuses on investigating the thermodynamic properties of tunable cells under related design parameters. The universal expression for the local entropy generation rate in such metamaterial systems is obtained considering the influence of rotation. A series of contrast schemes are established to describe the thermodynamic process and thermal energy distributions from the viewpoint of entropy analysis. Moreover, effects of design parameters on thermal dissipations and system irreversibility are investigated. In conclusion, more thermal dissipations and stronger thermodynamic processes occur in a system with larger conductivity ratios and rotation angles. This paper presents a detailed description of the thermodynamic properties of metamaterial tunable cells and provides reference for selecting appropriate design parameters on related positions to fabricate more efficient and energy-economical switchable TO devices.
\end{abstract}

Keywords: thermodynamic properties; entropy generation; tunable cell; metamaterial

\section{Introduction}

Innovations in the application of transformation optics (TO) [1] have been widely used to manipulate wave transformation in the fields of optics [2,3], electromagnetic [4,5], elastodynamics [6,7], and acoustics $[8,9]$ through artificial structures by mapping the coordinate transformations onto the spatial distributions of material properties. Especially, from the moment that the form invariance of heat diffusion equation was demonstrated in diffused fields [10], the aforementioned innovations have motivated investigations relative to heat transport leading to a number of significant findings on active control of thermal transfer using metamaterials. As far as the manipulation of heat transport can be regarded as regulating heat flux in specific media, the effective medium theory was employed to engineer thermal materials [11] for making anticipant novel achievements. Owing to experiments utilizing TO, a similar theory, named transformation thermodynamics [12], has been developed by taking advantage of the Jacobian matrix. From it, details of the spatial distributions of conductivities for designing 2D thermal cloaks and concentrators have been deduced. By using the method of arranging materials to create anisotropy in artificial structures, a number of schemes of 2D regular cloaks [13,14], 2D arbitrary shape cloaks [15], feeling cloak [16], thermal ground cloak [17], and 3D 
cloak [18] have been designed and experimented. In addition, a concept of response entropy [19] has been proposed to predict the cloaking performance under certain design parameters. Furthermore, the scattering-cancellation method [20-23], which is widely used in the field of electromagnetics, is also used to achieve thermal cloaks using natural materials. In addition, a kind of invisible sensor [23] has also been proposed based on the scattering-cancellation method, which could sense and camouflage in both thermal and DC fields simultaneously. Thermal harvesting can be also be realized by simultaneously expanding artificial regions and compressing concentrated regions [24]. Then, in order to achieve geometrically independent concentrators, a theoretical concept and a general design for a 3D concentrator [25] was proposed using natural conductive materials. Also, for achieving the compression function of heat flux, a new class of thermal metamaterial elements shaped in the form of "sensu" (fan) structures [26,27] were employed. On the basis of the design of thermal cloaks and concentrators, the camouflage was developed by controlling and reshaping transient thermal diffusion $[28,29]$. Furthermore, the concept of a thermal lens combined with anisotropic positive thermal conductivity was proposed for remote heating and cooling [30,31]. By considering form invariance after coordinate transformation in Laplace fields, two kinds of multifunctional TO devices were designed and fabricated. One was the multifunctional TO device, which could perform two functions in the same Laplace field (thermal field) using thermal diodes $[32,33]$. The other scheme could simultaneously perform two functions in different Laplace fields (thermal and electric) [34-36].

Among all the early studies related to the above, the goals are mainly related to the design and verification of novel TO devices with single or multiple functions. However, they are still far from practical application owing to the limitations of flexibly switching to different functions and fabricating large quantities of thermal metamaterials. In order to overcome these limitations, heat flux paths [37-41] with certain space rotations have been investigated recently. Related findings show that the bending of heat flux in multilayer artificial structures depends on geometrical azimuths, radial positions, material layer thicknesses, and thermal conductivity ratios [37-40]. By regulating related parameters, the conductive heat flux could be guided in a pre-determined direction [41], and in this regard, a metamaterial tunable cell, called thermal shifter, has been proposed [42]. Multiple functions can be performed and switched to flexibly by arranging several tunable cells on related positions based on pre-designed heat flux bending. Furthermore, an optimization-based design of heat flux manipulation was also proposed with such tunable cells [43]. However, the effect of irreversible heat loss could not be ignored as it leads to the merging of individual cells and rotations in one single cell, thus directly modifying the expected function. Hence, further investigations on the thermodynamic properties of tunable cells are needed to provide references in fabricating more efficient and energy-economical switchable TO devices.

In this paper, entropy generation analysis $[18,19,24,44,45]$ considering the rotation of material layers in tunable cells was introduced to investigate thermodynamic properties under multiple variables. A universal expression of local entropy generation rate in such a rotation system was proposed to describe the energy distributions in tunable cells. Furthermore, the temperature distributions and local entropy generation rates for contrast schemes were obtained to define the thermodynamic process. Finally, the thermal dissipations and system irreversibility were investigated by calculating the total entropy generation rate at varying thermal conductivity ratios and rotation angles.

\section{Theoretical Method and Geometrical Models}

\subsection{Rotation of Heat Flux Based on Transformation Optics}

The metamaterial tunable cell shown in Figure 1a is alternately composed of two kinds of thermal materials. Hence, heat flux conduction in such a metamaterial system changes because the components have different gradients of thermal conductivities. By taking into account the heat transfer in the series and parallel configurations of such a system, as given in [37-40], the tensors of heat conductivities in 
different directions in the 2D domain can be obtained through the effective medium approach [11], as follows:

$$
\kappa=\left(\begin{array}{cc}
\kappa_{x} & 0 \\
0 & \kappa_{y}
\end{array}\right)=\left(\begin{array}{cc}
\frac{\left(l_{\mathrm{A}}+l_{\mathrm{B}}\right) \kappa_{\mathrm{A}} \kappa_{\mathrm{B}}}{l_{\mathrm{A}} \kappa_{\mathrm{B}}+l_{\mathrm{B}} \kappa_{\mathrm{A}}} & 0 \\
0 & \frac{\kappa_{\mathrm{A}} l_{\mathrm{A}}+\kappa_{\mathrm{B}} l_{\mathrm{B}}}{l_{\mathrm{A}}+l_{\mathrm{B}}}
\end{array}\right) .
$$

where, $l_{\mathrm{A}}$ and $l_{\mathrm{B}}$ are the thicknesses $(\mathrm{m})$ of materials $\mathrm{A}$ and $\mathrm{B}$, and $\kappa_{\mathrm{A}}$ and $\kappa_{\mathrm{B}}$ denote the corresponding thermal conductivities $\left(\mathrm{W} \cdot \mathrm{m}^{-1} \cdot \mathrm{K}^{-1}\right)$.

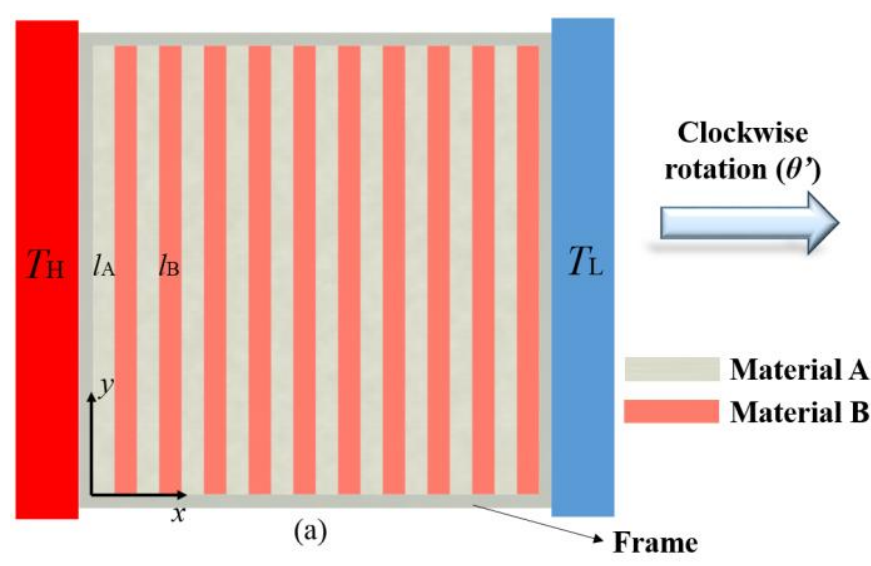

Figure 1. Schematic of the rotation process of the metamaterial tunable cell. (a) A single cell without rotation; (b) A clockwise rotated $\left(\theta^{\prime}\right)$ single cell.

The schematic of the transformation process is shown in Figure 1b. In order to achieve the function of regulating heat flux path and bending, the original domain $\Omega$ should be mapped onto the transformational domain $\Omega^{\prime}$ by rotating the entire system around the normal direction $(z)$. That is, the related process of coordinate transformation should allow the following conditions, where $\theta_{\text {ro }}$ denotes the rotational angle of the system:

$$
r^{\prime}=r, \theta^{\prime}=\theta+\theta_{\text {ro }}
$$

In order to manipulate the transformation process in the rotation system, the Jacobian matrix is employed to correlate the original and transformational domains, as given by Equation (3):

$$
\begin{aligned}
J & =\frac{\partial\left(x^{\prime}, y^{\prime}\right)}{\partial(x, y)}=\frac{\partial\left(x^{\prime}, y^{\prime}\right)}{\partial\left(r^{\prime}, \theta^{\prime}\right)} \frac{\partial\left(r^{\prime}, \theta^{\prime}\right)}{\partial(r, \theta)} \frac{\partial(r, \theta)}{\partial(x, y)} \\
& =\left(\begin{array}{ll}
\cos \theta^{\prime} & \sin \theta^{\prime} \\
-\sin \theta^{\prime} & \cos \theta^{\prime}
\end{array}\right) \operatorname{diag}\left(1, \frac{r^{\prime}}{r}\right)\left(\begin{array}{cc}
\cos \theta & \sin \theta \\
-\sin \theta & \cos \theta
\end{array}\right) .
\end{aligned}
$$

Taking Equation (2) into Equation (3), the Jacobian matrix for the transformation process is obtained:

$$
J=\left(\begin{array}{cc}
\cos \theta \cos \theta^{\prime}-\sin \theta \sin \theta^{\prime} & \sin \theta \cos \theta^{\prime}+\cos \theta \sin \theta^{\prime} \\
-\cos \theta \sin \theta^{\prime}-\sin \theta \cos \theta^{\prime} & -\sin \theta \sin \theta^{\prime}+\cos \theta \cos \theta^{\prime} e
\end{array}\right) .
$$

According to the Jacobian matrix, the effective thermal conductivity tensors in the transformational domain can be associated through the following expression:

$$
\kappa^{\prime}=\frac{J \kappa J^{\prime}}{\operatorname{det}(J)} .
$$

Taking Equation (4) into Equation (5), the effective thermal conductivity tensors in the transformational domain can be presented as: 


$$
\begin{gathered}
\kappa_{x^{\prime} x^{\prime}}=\kappa_{y^{\prime} y^{\prime}}=\frac{\kappa_{x}\left(\cos \theta \cos \theta^{\prime}-\sin \theta \sin \theta^{\prime}\right)^{2}+\kappa_{y}\left(\sin \theta \cos \theta^{\prime}+\cos \theta \sin \theta^{\prime}\right)^{2}}{\left(\cos \theta \cos \theta^{\prime}-\sin \theta \sin \theta^{\prime}\right)^{2}+\left(\sin \theta \cos \theta^{\prime}+\cos \theta \sin \theta^{\prime}\right)^{2}} \\
\kappa_{x^{\prime} y^{\prime}}=\kappa_{y^{\prime} x^{\prime}}=\frac{\left(-\kappa_{x}+\kappa_{y}\right)\left(\cos \theta \cos \theta^{\prime}-\sin \theta \sin \theta^{\prime}\right)\left(\sin \theta \cos \theta^{\prime}+\cos \theta \sin \theta^{\prime}\right)}{\left(\cos \theta \cos \theta^{\prime}-\sin \theta \sin \theta^{\prime}\right)^{2}+\left(\sin \theta \cos \theta^{\prime}+\cos \theta \sin \theta^{\prime}\right)^{2}} .
\end{gathered}
$$

Based on rotations above, the heat transfer process in the 2D transformational domain with the heat flux along the $x$ direction of the original domain can be observed with an additional second derivation of $\partial^{2} T / \partial x^{\prime} \partial y^{\prime}$, which was caused by the space rotation [39]:

$$
\rho^{\prime} c^{\prime} \frac{\partial T}{\partial t}=\nabla \cdot\left(\kappa_{x^{\prime} x^{\prime}} \nabla T+2 \kappa_{x^{\prime} y^{\prime}} \nabla T+\kappa_{y^{\prime} y^{\prime}} \nabla T\right) .
$$

where, $\rho^{\prime}, c^{\prime}$, and $T$ denote the efficient density $\left(\mathrm{kg} \cdot \mathrm{m}^{-3}\right)$, specific heat capacity $\left(\mathrm{J} \cdot \mathrm{K}^{-1} \cdot \mathrm{kg}^{-1}\right)$, and the transient temperature $(\mathrm{K})$ in the transformational domain. The constant temperature gradient $[37-40,42,43]$ $\left(\nabla T_{x}\right)$ along the $x$-direction of the original domain consists of two separate components, $\nabla T_{x^{\prime}}$ and $\nabla T_{y^{\prime}}$, in the transformation domain as a result of spatial rotations. That is, the transformation by Fourier's law becomes:

$$
\left(\begin{array}{l}
q_{x^{\prime}} \\
q_{y^{\prime}}
\end{array}\right)=-\left(\begin{array}{ll}
\kappa_{x^{\prime} x^{\prime}} & \kappa_{x^{\prime} y^{\prime}} \\
\kappa_{y^{\prime} y^{\prime}} & \kappa_{y^{\prime} y^{\prime}}
\end{array}\right)\left(\begin{array}{c}
\nabla T_{x^{\prime}} \\
\nabla T_{y^{\prime}}
\end{array}\right) .
$$

Taking Equations (6a,b), and (7) into Equation (8), the heat flux tensors in the $x^{\prime}$ and $y^{\prime}$ directions of the transformation domains are observed:

$$
\begin{aligned}
q_{x^{\prime}}= & -\frac{\kappa_{x}\left(\cos \theta \cos \theta^{\prime}-\sin \theta \sin \theta^{\prime}\right)^{2}+\kappa_{y}\left(\sin \theta \cos \theta^{\prime}+\cos \theta \sin \theta^{\prime}\right)^{2}}{\left(\cos \theta \cos \theta^{\prime}-\sin \theta \sin \theta^{\prime}\right)^{2}+\left(\sin \theta \cos \theta^{\prime}+\cos \theta \sin \theta^{\prime}\right)^{2}} \frac{\partial T}{\partial x^{\prime}} \\
& -\frac{\left(-\kappa_{x}+\kappa_{y}\right)\left(\cos \theta \cos \theta^{\prime}-\sin \theta \sin \theta^{\prime}\right)\left(\sin \theta \cos \theta^{\prime}+\cos \theta \sin \theta^{\prime}\right)}{\left(\cos \theta \cos \theta^{\prime}-\sin \theta \sin \theta^{\prime}\right)^{2}+\left(\sin \theta \cos \theta^{\prime}+\cos \theta \sin \theta^{\prime}\right)^{2}} \frac{\partial T}{\partial y^{\prime \prime}} \\
q_{y^{\prime}}= & -\frac{\left(-\kappa_{x}+\kappa_{y}\right)\left(\cos \theta \cos \theta^{\prime}-\sin \theta \sin \theta^{\prime}\right)\left(\sin \theta \cos \theta^{\prime}+\cos \theta \sin \theta^{\prime}\right)}{\left(\cos \theta \cos \theta^{\prime}-\sin \theta \sin \theta^{\prime}\right)^{2}+\left(\sin \theta \cos \theta^{\prime}+\cos \theta \sin \theta^{\prime}\right)^{2}} \frac{\partial T}{\partial x^{\prime}} \\
& -\frac{\kappa_{x}\left(\cos \theta \cos \theta^{\prime}-\sin \theta \sin \theta^{\prime}\right)^{2}+\kappa_{y}\left(\sin \theta \cos \theta^{\prime}+\cos \theta \sin \theta^{\prime}\right)^{2}}{\left(\cos \theta \cos \theta^{\prime}-\sin \theta \sin \theta^{\prime}\right)^{2}+\left(\sin \theta \cos \theta^{\prime}+\cos \theta \sin \theta^{\prime}\right)^{2}} \frac{\partial T}{\partial y^{\prime}} .
\end{aligned}
$$

In order to investigate the thermodynamic performances of the metamaterial tunable cell, entropy generation analysis $[18,19,24,44,45]$ is introduced to define the thermal dissipations of different schemes with varying rotations and selected materials. The volumetric local entropy generation rate for such systems in a transformed 2D domain $[19,24,44,45]$ can be observed using the Fourier transform:

$$
\dot{S}_{g e n}^{\prime \prime \prime}=\frac{\partial}{\partial x^{\prime}}\left(\frac{q_{x^{\prime}}}{T}\right)+\frac{\partial}{\partial y^{\prime}}\left(\frac{q_{y^{\prime}}}{T}\right)-\frac{1}{T} \dot{q}
$$

where, $\dot{S}_{\text {gen }}^{\prime \prime \prime}$ is the volumetric local entropy generation rate $\left(\mathrm{W} \cdot \mathrm{m}^{-3} \cdot \mathrm{K}^{-1}\right) \cdot \dot{q}$ denotes the heat flux of source.

Taking Equation $(9 a, b)$ into Equation (10), the volumetric local entropy generation rate in the transformation domain is observed:

$$
\begin{aligned}
\dot{S}_{\text {gen }}^{\prime \prime \prime}= & \left(\frac{\kappa_{x}\left(\cos \theta \cos \theta^{\prime}-\sin \theta \sin \theta^{\prime}\right)^{2}}{T^{2}\left(\left(\cos \theta \cos \theta^{\prime}-\sin \theta \sin \theta^{\prime}\right)^{2}+\left(\sin \theta \cos \theta^{\prime}+\cos \theta \sin \theta^{\prime}\right)^{2}\right)}\right)\left(\left(\frac{\partial T}{\partial x^{\prime}}\right)^{2}+\left(\frac{\partial T}{\partial y^{\prime}}\right)^{2}\right) \\
& +\frac{2\left(-\kappa_{x}+\kappa_{y}\right)\left(\cos \theta \cos \theta^{\prime}-\sin \theta \sin \theta^{\prime}\right)\left(\sin \theta \cos \theta^{\prime}+\cos \theta \sin \theta^{\prime}\right)}{T^{2}\left(\left(\cos \theta \cos \theta^{\prime}-\sin \theta \sin \theta^{\prime}\right)^{2}+\left(\sin \theta \cos \theta^{\prime}+\cos \theta \sin \theta^{\prime}\right)^{2}\right)} \frac{\partial T}{\partial x^{\prime}} \cdot \frac{\partial T}{\partial y^{\prime}} .
\end{aligned}
$$


Considering the dividing ratio of the thermal conductivities in different directions [40]:

$$
\frac{\kappa_{y}}{\kappa_{x}}=\frac{\frac{\kappa_{\mathrm{A}} l_{\mathrm{A}}+\kappa_{\mathrm{B}} l_{\mathrm{B}}}{l_{\mathrm{A}}+l_{\mathrm{B}}}}{\frac{\left(l_{\mathrm{A}}+l_{\mathrm{B}}\right) \kappa_{\mathrm{A}} \kappa_{\mathrm{B}}}{l_{\mathrm{A}} \kappa_{\mathrm{B}}+l_{\mathrm{B}} \kappa_{\mathrm{A}}}}=1+\frac{l_{\mathrm{A}} l_{\mathrm{B}}}{\left(l_{\mathrm{A}}+l_{\mathrm{B}}\right)^{2}} \cdot \frac{\left(\kappa_{\mathrm{A}}-\kappa_{\mathrm{B}}\right)^{2}}{\kappa_{\mathrm{A}} \kappa_{B}}=1+\varepsilon
$$

In order to simplify Equation (11), we introduce a new variable $\sigma=\left(\sin \theta \cos \theta^{\prime}+\cos \theta \sin \theta^{\prime}\right) /$ $\left(\cos \theta \cos \theta^{\prime}-\sin \theta \sin \theta^{\prime}\right)$, which allows to characterize the relations of the rotation between the original and transformational domains. The form of Equation (11) can be written as:

$$
\dot{S}_{\text {gen }}^{\prime \prime \prime}=\frac{\kappa_{x}}{T^{2}\left(1+\sigma^{2}\right)}\left(\left(1+(1+\varepsilon) \sigma^{2}\right)\left(\left(\frac{\partial T}{\partial x^{\prime}}\right)^{2}+\left(\frac{\partial T}{\partial y^{\prime}}\right)^{2}\right)+2 \varepsilon \sigma \frac{\partial T}{\partial x^{\prime}} \cdot \frac{\partial T}{\partial y^{\prime}}\right) .
$$

It can be seen that Equation $(9 a, b)$ would be in accordance with the findings in [37-39], after applying the constant temperature gradients along the $x$ directions and no rotations in the original domains. Furthermore, the local entropy generation rate of such a system could be achieved with the thermal flux above by Equation (10), which was widely used in investigating thermodynamic performance $[19,24,44,45]$. Hence, the accuracy of the mathematical derivations could be guaranteed.

\subsection{Description of Tunable Cell Scheme}

To regulate heat flux vectors in pre-determined paths, the creation of the schematic of the metamaterial tunable cell with conductivity gradients in the function regions should be based on the rotation process described above. Additionally, based on the effective medium theory [11], two kinds of thermal materials with large differences in conductivities are alternately combined to create a field of conductivity gradients. Once the selected materials are combined in parallel and in series under corresponding rotations, the heat flux bends [37-41] following the pre-designed direction. That means that the degree of rotation of the entire system and related material properties directly affect heat flux bending. The thermodynamic properties are also altered by changing variables as confirmed by Equation (13), following the second law of thermodynamics.

In examining the thermodynamic properties of the tunable cell under varying rotation angles and conductivity ratios, schemes of tunable cells with several polydimethylsiloxane (PDMS, $\left.\kappa_{\mathrm{A}}=0.15 \mathrm{~W} \cdot \mathrm{m}^{-3} \cdot \mathrm{K}^{-1}\right)$ and copper $\left(\kappa_{\mathrm{B}}=398 \mathrm{~W} \cdot \mathrm{m}^{-3} \cdot \mathrm{K}^{-1}\right)$ layers were proposed to satisfy the requirements of the conductivity gradient. As to the rotation relativity between the original and transformational domains, the initial rotation angle was set as $0^{\circ}$ in the original domain, i.e., the initial scheme has no rotation $\left(\theta=0^{\circ}\right)$, as shown in Figure $1 \mathrm{a}$, and the rotation angle in a transformed scheme was designated $\theta_{\text {ro }}\left(\theta^{\prime}=\theta_{\text {ro }}\right)$. Hence, three transformed schemes with rotation angles of $30^{\circ}$, $45^{\circ}$, and $60^{\circ}$ were respectively created. As [37-41] pointed out, for all transformed schemes, larger anisotropy led to larger heat flux bending in the tunable cells. Hence, the thicknesses of PDMS and copper layers were made uniform, i.e., $l_{\mathrm{A}}=l_{\mathrm{B}}=0.0015 \mathrm{~mm}$, in order to obtain the largest anisotropy in thermal conductivities based on Equation (12). And the height of each layer was set at $0.03 \mathrm{~m}$. Under the related conditions above, the local entropy generation rate of Equation (13) can be written as:

$$
\dot{S}_{\text {gen }}^{\prime \prime \prime}=\frac{n \kappa_{A} \cos ^{2} \theta_{\mathrm{ro}}}{(1+n) T^{2}}\left(\left(1+\frac{(n+1)^{2} \tan ^{2} \theta_{\mathrm{ro}}}{4 n}\right)\left(\left(\frac{\partial T}{\partial x^{\prime}}\right)^{2}+\left(\frac{\partial T}{\partial y^{\prime}}\right)^{2}\right)+\frac{(n-1)^{2} \tan \theta_{\mathrm{ro}}}{2 n} \frac{\partial T}{\partial x^{\prime}} \cdot \frac{\partial T}{\partial y^{\prime}}\right) .
$$

where, $\sigma=\tan \theta_{r o}, n$ denotes the ratio of materials $\mathrm{B}$ and $\mathrm{A}$, i.e., $n=\kappa_{\mathrm{B}} / \kappa_{\mathrm{A}}$.

The initial scheme without rotation was made of 10 PDMS and 10 copper layers alternately combined and forming a square plate with a side length of $0.03 \mathrm{~m}$. Then, to achieve significant bending effects, the entire scheme was set into a copper square frame with a thickness of $0.001 \mathrm{~m}$ and an outer side length of $0.032 \mathrm{~m}$. The other three schemes were also set into a similar square copper frame but with material layers oriented at different rotation angles. The left boundary of the frame 
was set as a high temperature boundary with a constant temperature of $T_{\mathrm{H}}=353 \mathrm{~K}$, and the right boundary was fixed to a constant low temperature of $T_{\mathrm{L}}=293 \mathrm{~K}$. In addition, the ambient temperature was kept at $293 \mathrm{~K}$. Finally, as a means to investigate the thermodynamic properties of the schemes, numerical simulations based on the finite volume method were utilized through ANSYS Fluent. Furthermore, the related mesh independency analysis for the rotation schemes and the validations of the obtained results can be found in the Sections S1 and S2 of the "Supplementary Materials", respectively. In addition, the obtained derivations can be also extended to $3 \mathrm{D}$ thermal metamaterials, the details can be found in the Section S3 of the "Supplementary Materials".

\section{Thermodynamic Performance of Tunable Cell Schemes}

\subsection{Properties of Temperature Distributions and Heat Flux Bending}

Accordingly, the rotation transformation process, distributions of temperature fields, and heat flux of the schemes at time $t=150 \mathrm{~s}$ were observed under the temperature conditions mentioned above.

It can be observed from Figure 2 that an upward heat flux bending occurred in all schemes owing to clockwise rotations, indicating that the direction of heat flux bending can be adjusted by regulating the direction of the rotation angles. Meanwhile, the declination angles in heat flux were $59.67^{\circ}, 44.85^{\circ}$, and $29.76^{\circ}$ for each scheme shown in Figure 2, which were in accordance with those observed by Equation (9) given in [37]. Moreover, heat flux bending increased as the magnitude of the rotation angle decreased. Owing to the large ratio of thermal conductivities of copper and PDMS, the anisotropies in the schemes had covariant enhancements, i.e., the values calculated by Equation (12) approached infinity, leading to expressions of heat flux bending given in [37-40] close to $\tan ^{-1}\left(\cot \theta_{\text {ro }}\right)$. Hence, heat flux bending was approaching $90^{\circ}-\theta_{\text {ro }}$. In addition, the heat fluxes approaching the left and right sides of the frame regions are not orthogonal to the $y$-direction, therefore causing rotations of adjacent metamaterial layers. Meanwhile, path variations in the downward bending of heat flux in the surrounding frame reduced with increasing magnitudes of the rotation angle. In other words, larger rotation angles led to smaller distortions in heat flux paths in the surroundings. This condition would help in the transition of heat flux directions between adjacent tunable cells containing latent applications [42,43]. Furthermore, the distributions of temperature fields have covariant transformations with the rotation heat flux. These indicate that the perturbations of the temperature isotherms increased with increasing rotation angles. The temperature fields were also more uniform as the arrangements of materials were more parallel to the directions of heat flow, i.e., the heat transfer distance in the parallel materials (along $x$ direction) of PDMS layers increased significantly.

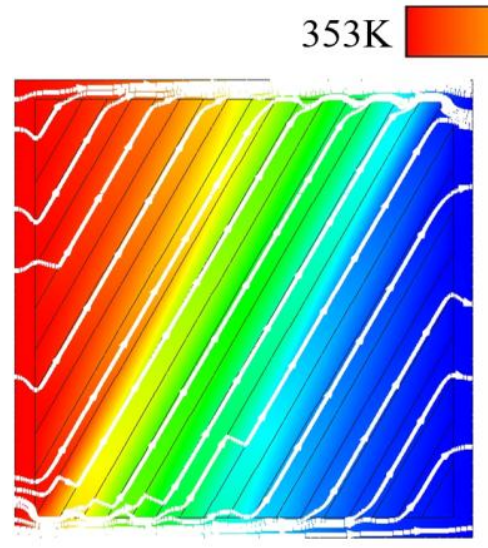

(a)

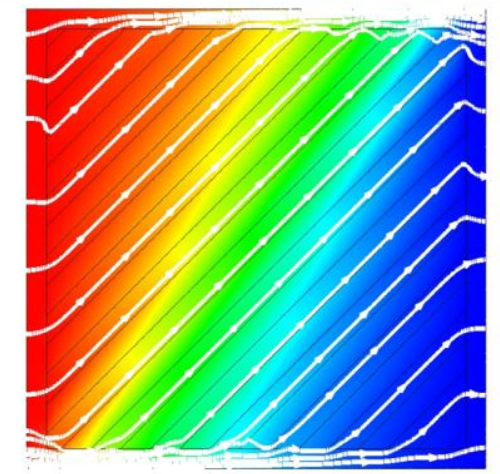

(b)
$293 \mathrm{~K}$

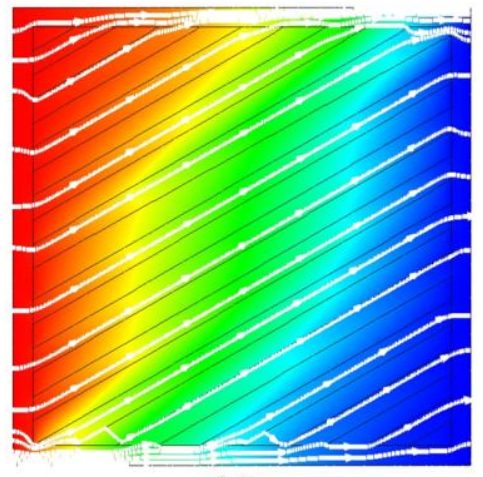

(c)

Figure 2. Distributions of temperature fields and related heat flux (white lines) of contrast schemes. (a) Scheme of rotating $30^{\circ}$; (b) Scheme of rotating $45^{\circ}$; (c) Scheme of rotating $60^{\circ}$. 


\subsection{Analysis of Entropy Generation}

To further investigate the thermodynamic performance in adjacent layers, the temperature distributions in the central lines along the $x$-direction $(y=0.015 \mathrm{~m})$ shown in Figure 3 were selected. Temperature distributions on the measured lines are shown in Figure 3. It is evident that temperature distributions are more uniform along the $x$-direction with increasing rotation angles, i.e., deviations in temperatures between adjacent testing points were smaller. The upper right inset illustrates the local entropy generation rates along the central lines. It can be seen that the highest values in a separate scheme were observed in the copper layers, and the local entropy rates in the PDMS layers approached zero. That is, stronger thermodynamic processes occurred in the copper layer because of its higher thermal conductivity. Hence, the distributions of thermal energy in the PDMS layers were far more random than those in the copper layers. In addition, stronger thermodynamic processes occurred with increasing rotation angles as schemes with larger rotation angles were also found to have larger perturbations in local entropy rates. These indicate that thermal energy disturbances were reduced and the thermodynamic process is enhanced by increasing rotation angles. Thus, this latter increase forced a smoother heat transfer, i.e., smaller temperature deviations between adjacent layers along the central lines.

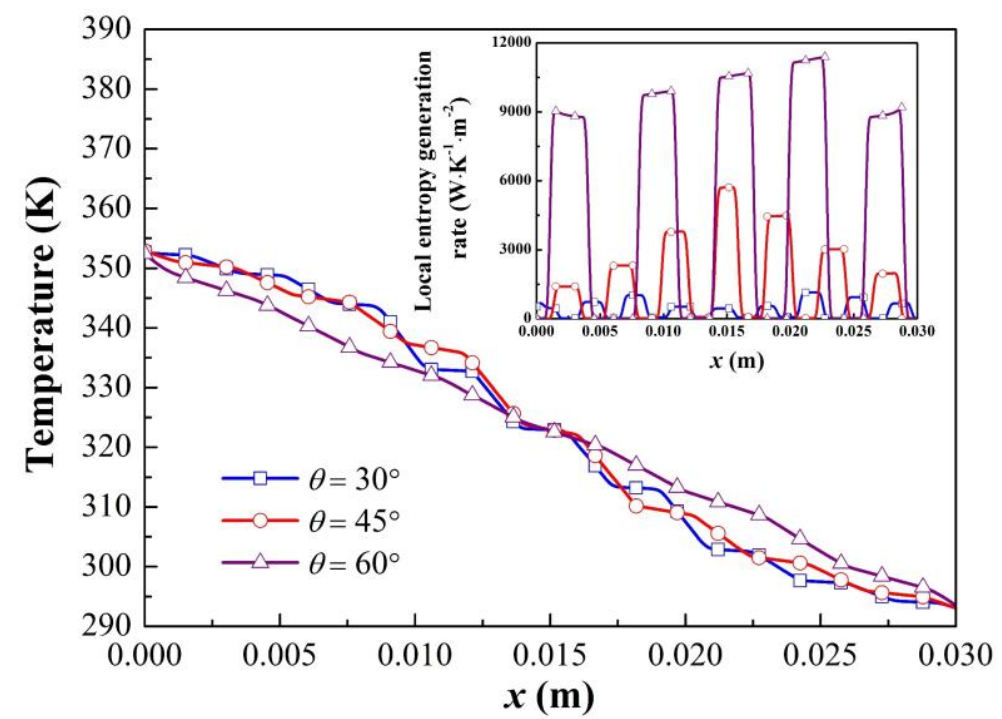

Figure 3. Temperature distributions along $x$ direction on the central line of $y=0.015 \mathrm{~m}$ at $150 \mathrm{~s}$ of the three contrast schemes. The upper inset denotes the local entropy generation rate on the measured line.

To extend the examination of the local entropy generation rates of metamaterial tunable cells, the distributions of these generation rates are illustrated in Figure 4. The local entropy generation rates of the initial scheme without rotation are also presented to make a rational comparison. As shown in Figure $4 \mathrm{a}$, the distributions of local entropy generation rates in the material layers approached zero, followed a uniform trend, and appeared as "ladders" owing to the conductivity gradients. Moreover, the local entropy generation rates on the inside top and bottom boundaries of the frames were noticeably higher than those in the material layers. That is, the inside top and bottom boundaries of the frames were directly integrated with the material layers, resulting in large differences in entropy generation rates and anisotropies in the conductivities between adjacent boundaries. Hence, the perturbations of thermal energy were more intense at frame boundaries than those in the metamaterial layers. Figure $4 \mathrm{~b}-\mathrm{d}$ present the local entropy generation rates of the other three schemes with rotation angles of $30^{\circ}, 45^{\circ}$, and $60^{\circ}$, respectively. It can be seen that local entropy generation rates gradually increased with increasing rotation angles, which means that stronger thermodynamic processes occurred with larger rotation angles. Hence, the thermal energy distribution was more 
orderly. Furthermore, the parallel distance components of heat transfer along the $x$-direction in both layers increased as a result of increasing rotation angles. Consequently, this directly led to more irreversible heat losses and thermal dissipations in the material layers, which was similarly reflected in the distributions of local entropy generation rates. Moreover, the response of the copper layers to changing rotations was more intense than those in PDMS layers. As such, the varying rotations significantly affected the thermodynamic processes in the material layers with higher conductivities. Conversely, the perturbations of thermal energy were not apparent in materials with lower conductivities. Therefore, more dissipations occurred in the copper layers with increasing rotation angles, which forced the temperatures at the interface of the two material layers to be more similar, as indicated in Figure 3.

(a)

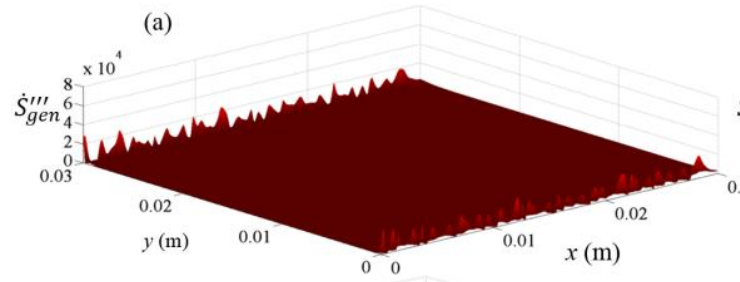

(c)

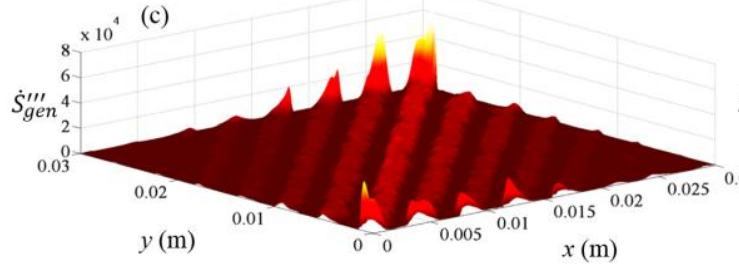

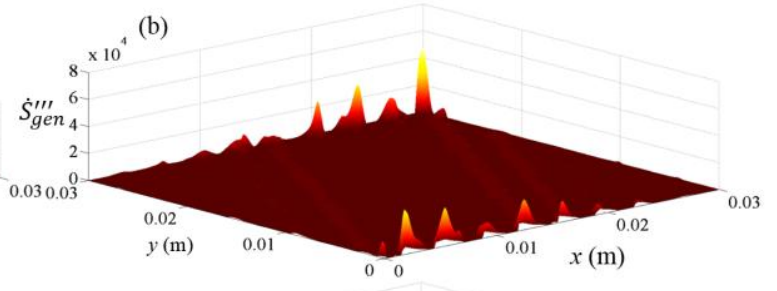

(d)

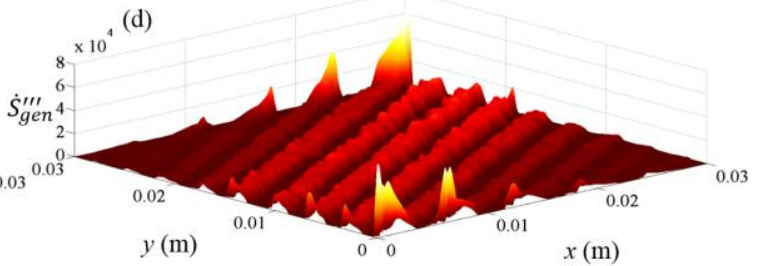

Figure 4. Distributions of local entropy generation rates of the entire systems for each scheme. (a) Initial scheme without rotation; (b) Scheme with a rotation angle of $30^{\circ}$; (c) Scheme with a rotation angle of $45^{\circ}$; (d) Scheme with a rotation angle of $60^{\circ}$.

In order to acquire a comprehensive description of the thermodynamic analysis of the metamaterial tunable cell by taking into consideration the multiple parameters of rotation angle $\left(\theta_{\text {ro }}\right)$ and ratio of material conductivities $(n)$, five additional initial schemes with different ratios of material conductivities $(n)$ were created. Having observed that bending heat flux depended on creating high anisotropy in the tunable cells, obviously, the material with low conductivity is irreplaceable. As [27] pointed out, PDMS is a perfect material for fabricating thermal metamaterial as its industrially preferable and has lower conductivity. Hence, in this study, PDMS continued to be used (material A) as in the three original schemes above. However, the other material (material B) was adjusted to achieve different ratios of material conductivities. Also, in order to make reasonable comparisons, the five new schemes were created by separately employing PDMS as material A and the five other materials as material $\mathrm{B}$ with conductivities of $300,150,75,15$, and $0.15 \mathrm{~W} \cdot \mathrm{m}^{-1} \cdot \mathrm{K}^{-1}$, which correspond to the ratios of material conductivities of 2000,1000,500,100, and 1 . As for the thermodynamic analysis of the entire system under varying clockwise rotations angles, the equilibrium total entropy generation rates were observed to represent the total dissipation by integrating Equation (14) for the whole metamaterial domain:

$$
\dot{S}_{g e n}=\iint \dot{S}_{g e n}^{\prime \prime \prime} d A
$$

Figure 5 illustrates the variations in total entropy generation rates with the rotation angles $\left(\theta_{\mathrm{ro}}\right)$ when the ratios of the conductivities were fixed. The figure indicates that the equilibrium total entropy generation rates increased with increasing conductivity ratios. It is seen that with any rotation angle, if $n=1$, the equilibrium total entropy generation rates were zero, which indicate that there was no conductivity gradient and the system was homogeneous. Hence, no effects on bending heat flux could be observed. The symmetry of the rotation process also affected the values centered on $\theta_{\text {ro }}=90^{\circ}$. 
The values first increased and then declined as soon as the conductivity ratio was fixed. Moreover, the values varied nonlinearly in the ranges of $0^{\circ}-90^{\circ}$ and $90^{\circ}-180^{\circ}$. Before $\theta_{\text {ro }}$ approached $30^{\circ}$, the rate of change (slope) of the rotation angles gradually increased with increasing clockwise rotation angles. However, with $\theta_{\text {ro }}$ in the range of $30^{\circ}-90^{\circ}$, the slope rapidly increased. The varying trends when $\theta_{\text {ro }}$ was within the range of $90^{\circ}-180^{\circ}$ were different from those when $\theta_{\text {ro }}$ was within the range of $0^{\circ}-90^{\circ}$, owing to symmetry. That is, thermal dissipations and system irreversibility increased with increasing rotation angles in the range of $0^{\circ}-90^{\circ}$, owing to the increasing parallel distance component, which resulted in more irreversible heat loss. Moreover, the minimum values were obtained at $\theta_{\text {ro }}=0^{\circ}$ and $\theta_{\mathrm{ro}}=180^{\circ}$, when the system was without rotation. However, they are not zero, owing to the anisotropy in the system composed of two materials.

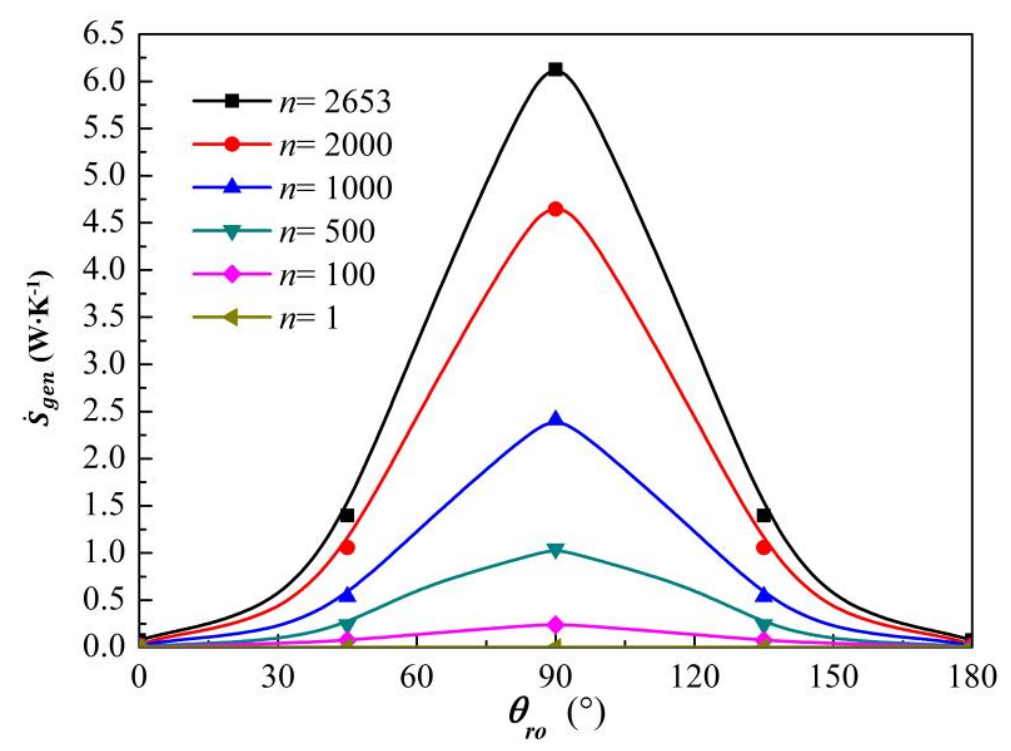

Figure 5. The variation of equilibrium total entropy generation rate versus $\theta_{\text {ro }}$ at fixed $n$.

Figure 6 illustrates the variations in total entropy generation rates with the ratios of conductivities when the rotation angles were fixed. It is evident that the total entropy generation rate increased with increasing rotation angles which agrees with that obtained in Figure 5. The slopes of total entropy generation also increased significantly with increasing clockwise rotation angles. Moreover, in all of the schemes, the increase in values were all linear once the rotation angles $\left(\theta_{\mathrm{ro}}\right)$ were fixed. This means that the thermal dissipations and system irreversibility increased with increasing conductivity ratios caused by the increasing anisotropy in the system. These increases led to more irreversible heat losses than in a system with smaller conductivity ratios. In addition, the perturbations in the total entropy generation rate decreased rapidly, indicating that large rotation angles contributed to the enhancement of the thermodynamic process.

It can be seen from Figures 5 and 6 that thermal dissipations and system irreversibility increased with increasing total entropy generation rates because of the large conductivity ratios and the increasing clockwise rotation angles in the range of $0^{\circ}-90^{\circ}$ (or declining clockwise rotation angles in the range of $\left.90^{\circ}-180^{\circ}\right)$. Hence, more thermal losses would occur in such schemes. Considering that applications in fabricating TO thermal devices employing tunable cells are latent, it is important that heat flux bending be controlled and regulated to achieve related functions by adjusting the rotation angles to fit the values of a single cell. However, the irreversible heat loss corresponding to different schemes also changed significantly with varying rotation angles. Hence, regulating the ratios of conductivities would be advantageous to reduce thermal dissipations and design more efficient and energy-economical TO devices. 


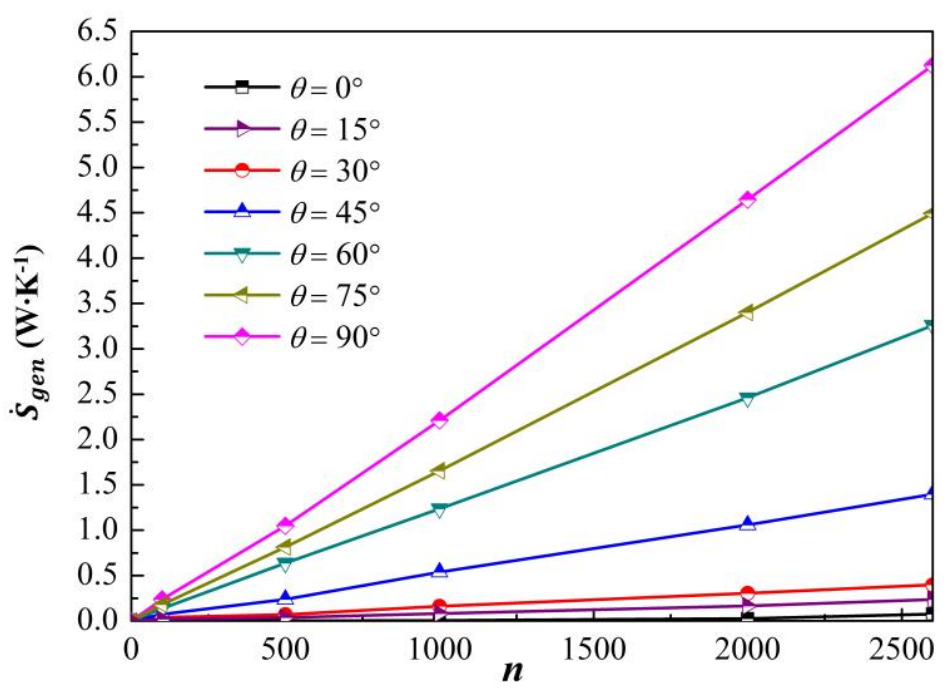

Figure 6. The variation of equilibrium total entropy generation rate versus $n$ at fixed $\theta_{\text {ro. }}$.

\section{Conclusions}

In this paper, we have investigated the thermodynamic processes of metamaterial tunable cells considering the influences of conductivity ratios and rotation angles. An expression for local entropy generation rate in such rotational systems has been proposed. According to the achieved results, the following can be concluded:

(1) The universal expression for local entropy generation rate in such a rotational system has been deduced by considering many rotation angles in the initial domain. The deduced expression contributed to a better understanding of the thermodynamic performances of functionally graded materials based on transformation optics.

(2) The local entropy generation rates of metamaterial tunable cells have been obtained. With increasing clockwise rotation angles, the local entropy generation rate increased accordingly, owing to the extensions of parallel distance components of the heat transfer process. However, it significantly increased in the layers with higher conductivities where stronger thermodynamic processes occurred.

(3) The total entropy generation rates of the entire system have been investigated. The values increased linearly with the increasing conductivity ratios. In addition, the highest values occurred when $\theta_{\text {ro }}=90^{\circ}$, i.e., the arrangement of materials was parallel to the $x$-direction; the minimum value occurred when $\theta_{\text {ro }}=0^{\circ}$, i.e., the schemes were without rotations.

(4) The thermal dissipations and system irreversibility would be enhanced with increasing conductivity ratio of the tunable cell scheme and clockwise rotation angles in the range of $0^{\circ}-90^{\circ}$ (or with reducing clockwise rotation angles in the range of $90^{\circ}-180^{\circ}$ ). Hence, the thermal energy distributions could be regulated by adjusting $n$ and $\theta_{\mathrm{ro}}$, and multifunctional TO devices can be easily fabricated via employing tunable cells.

The findings obtained in this paper will provide reference for investigating the thermodynamic performance of such rotation systems. Thus, the thermal dissipations and system irreversibility of different tunable cell schemes will also be considered in fabricating novel thermal devices by employing and assembling a certain amount of such tunable cells with different design parameters. By regulating the thermodynamic performances of the system investigated in this research, many latent, efficient, and energy-economical switchable TO devices will be explored and designed. That is, different functions including cloaking, concentrating, and rotating can be achieved in certain regions of one system, owing to the varying numbers and arrangements of such tunable cells. In addition, 
optimal performance can be obtained during the processes of switchover function by considering the thermodynamic performances inside such systems. Moreover, the findings will also provide reference for studying the transport losses of such TO devices in other fields.

Supplementary Materials: The following are available online at www.mdpi.com/1099-4300/19/10/538/s1, Section S1: Mesh independency analysis for the rotation schemes, Section S2: Validations of the obtained results, Section S3: Extensive derivation of 3D thermal metamaterials.

Acknowledgments: This work is supported by the National Natural Science Foundation of China (Grant Nos. 51776050 and 51536001).

Author Contributions: Guoqiang Xu and Haochun Zhang conceived and designed the research; Guoqiang Xu and Xiu Zhang conducted the calculation and analyzed the data; Yan Jin contributed analysis tools; Guoqiang Xu wrote the paper, with the assistance of Haochun Zhang.

Conflicts of Interest: The authors declare no conflict of interest.

\section{References}

1. Pendry, J.B.; Shurig, D.; Smith, D.R. Controlling electromagnetic fields. Science 2006, 312, $1780-1782$. [CrossRef] [PubMed]

2. Valentine, J.; Li, J.; Zentgraf, T.; Bartal, G.; Zhang, X. An optical cloak made of dielectrics. Nat. Mater. 2009, 8, 568-571. [CrossRef] [PubMed]

3. Ni, X.; Wong, Z.J.; Mrejen, M.; Wang, Y.; Zhang, X. An ultrathin invisibility skin cloak for visible light. Science 2015, 349, 1310-1314. [CrossRef] [PubMed]

4. Schurig, D.; Mock, J.J.; Justice, B.J. Metamaterial electromagnetic cloak at microwave frequencies. Science 2006, 314, 977-980. [CrossRef] [PubMed]

5. Gömöry, F.; Solovyov, M.; Šouc, J.; Navau, C.; Prat-Camps, J.; Sanchez, A. Experimental realization of a magnetic cloak. Science 2012, 335, 1466-1468. [CrossRef] [PubMed]

6. Bückmann, T.; Thiel, M.; Kadic, M.; Schittny, R.; Wegener, M. An elasto-mechanical unfeelability cloak made of pentamode metamaterials. Nat. Commun. 2014, 5. [CrossRef] [PubMed]

7. Kadic, M.; Bückmann, T.; Schittny, R.; Gumbsch, P.; Wegener, M. Pentamode metamaterials with independently tailored bulk modulus and mass density. Phys. Rev. Appl. 2014, 2. [CrossRef]

8. Hu, W.; Fan, Y.; Ji, P.; Yang, J. An experimental acoustic cloak for generating virtual images. J. Appl. Phys. 2013, 113, 24911. [CrossRef]

9. Li, Q.; Vipperman, J.S. Two-dimensional acoustic cloaks of arbitrary shape with layered structure based on transformation acoustics. Appl. Phys. Lett. 2014, 105, 101906. [CrossRef]

10. Fan, C.Z.; Gao, Y.; Huang, J.P. Shaped graded materials with an apparent negative thermal conductivity. Appl. Phys. Lett. 2008, 92, 251907. [CrossRef]

11. Narayana, S.; Sato, Y. Heat flux manipulation with engineered thermal materials. Phys. Rev. Lett. 2012, 108, 214303. [CrossRef] [PubMed]

12. Guenneau, S.; Amra, C.; Veynante, D. Transformation thermodynamics: Cloaking and concentrating heat flux. Opt. Express 2012, 20, 8207-8218. [CrossRef] [PubMed]

13. Schittny, R.; Kadic, M.; Guenneau, S.; Wegener, M. Experiments on transformation thermodynamics: Molding the flow of heat. Phys. Rev. Lett. 2013, 110, 195901. [CrossRef] [PubMed]

14. Ma, Y.; Lan, L.; Jiang, W.; Sun, F.; He, S. A transient thermal cloak experimentally realized through a rescaled diffusion equation with anisotropic thermal diffusivity. NPG Asia Mater. 2013, 5. [CrossRef]

15. Yang, T.; Huang, L.; Chen, F.; Xu, W. Heat flux and temperature field cloaks for arbitrarily shaped objects. J. Phys. D Appl. Phys. 2013, 46, 305102. [CrossRef]

16. Shen, X.Y.; Huang, J.P. Thermally hiding an object inside a cloak with feeling. Int. J. Heat Mass Tranf. 2014, 78. [CrossRef]

17. Yang, T.; Wu, Q.; Xu, W.; Liu, D.; Huang, L.; Chen, F. A thermal ground cloak. Phys. Lett. A 2016, 380, 965-969. [CrossRef]

18. Xu, G.Q.; Zhang, H.C. A concept of heat dissipation coefficient for thermal cloak based on entropy generation approach. AIP Adv. 2016, 6, 95107. [CrossRef] 
19. Xu, G.Q.; Zhang, H.C.; Zou, Q.; Jin, Y. Predicting and analyzing interaction of the thermal cloaking performance through response surface method. Int. J. Heat Mass Tranf. 2017, 109, 746-754. [CrossRef]

20. Han, T.; Yuan, T.; Li, B.; Qiu, C.W. Homogeneous thermal cloak with constant conductivity and tunable heat localization. Sci. Rep. 2013, 3. [CrossRef] [PubMed]

21. Han, T.; Bai, X.; Gao, D.; Thong, J.T.L.; Li, B.; Qiu, C.W. Experimental demonstration of a bilayer thermal cloak. Phys. Rev. Lett. 2014, 112, 54302. [CrossRef] [PubMed]

22. Nguyen, D.M.; Xu, H.; Zhang, Y.; Zhang, B. Active thermal cloak. Appl. Phys. Lett. 2015, 107, 121901. [CrossRef]

23. Yang, T.; Bai, X.; Gao, D.; Wu, L.; Li, B.; Thong, J.T.L.; Qiu, C.W. Invisible sensors: Simultaneous sensing and camouflaging in multiphysical fields. Adv. Mater. 2015, 27, 7752-7758. [CrossRef] [PubMed]

24. Xu, G.Q.; Zhang, H.C.; Zou, Q.; Xie, M.; Jin, Y. Forecast of thermal harvesting performance under multi-parameter interaction with response surface methodology. Int. J. Heat Mass Tranf. 2017, 115, 682-693. [CrossRef]

25. Han, T.; Zhao, J.; Yuan, T.; Lei, D.Y.; Li, B.; Qiu, C.W. Theoretical realization of an ultra-efficient thermal energy harvesting cell made of natural materials. Energy Environ. Sci. 2013, 6, 3537-3541. [CrossRef]

26. Han, T.; Bai, X.; Liu, D.; Gao, D.; Li, B.; Thong, J.T.L.; Qiu, C.W. Manipulating steady heat conduction by sensu-shaped thermal metamaterials. Sci. Rep. 2015, 5, 10242. [CrossRef] [PubMed]

27. Chen, F.; Lei, D.Y. Experimental realization of extreme heat flux concentration with easy-to-make thermal metamaterials. Sci. Rep. 2015, 5, 11552. [CrossRef] [PubMed]

28. He, X.; Wu, L. Illusion thermodynamics: A camouflage technique changing an object into another one with arbitrary cross section. Appl. Phys. Lett. 2014, 105, 221904. [CrossRef]

29. Yang, T.Z.; Su, Y.; Xu, W.; Yang, X.D. Transient thermal camouflage and heat signature control. Appl. Phys. Lett. 2016, 109, 121905. [CrossRef]

30. Liu, Y.; Sun, F.; He, S. Novel thermal lens for remote heating/cooling designed with transformation optics. Opt. Express 2016, 24, 5683-5692. [CrossRef] [PubMed]

31. Sun, F.; He, S. Remote cooling by a novel thermal lens with anisotropic positive thermal conductivity. Sci. Rep. 2017, 7, 40949. [CrossRef] [PubMed]

32. Li, Y.; Shen, X.; Wu, Z.; Huang, J.; Chen, Y.; Yi, Y.; Huang, J.P. Temperature-dependent transformation thermotics: From switchable thermal cloaks to macroscopic thermal diodes. Phys. Rev. Lett. 2015, 115, 195503. [CrossRef] [PubMed]

33. Shen, X.; Li, Y.; Jiang, C.; Huang, J.P. Temperature trapping: Energy-free maintenance of constant temperatures as ambient temperature gradients change. Phys. Rev. Lett. 2016, 117, 55501. [CrossRef] [PubMed]

34. Ma, Y.; Liu, Y.; Raza, M.; Wang, Y.; He, S. Experimental demonstration of a multiphysics cloak: Manipulating heat flux and electric current simultaneously. Phys. Rev. Lett. 2014, 113, 205501. [CrossRef] [PubMed]

35. Lan, C.; Bi, K.; Fu, X.; Li, B.; Zhou, J. Bifunctional metamaterials with simultaneous and independent manipulation of thermal and electric fields. Opt. Express 2016, 24, 23072-23080. [CrossRef] [PubMed]

36. Han, T.; Qiu, C.W. Transformation Laplacian metamaterials: Recent advances in manipulating thermal and dc fields. J. Opt. 2016, 18, 44003. [CrossRef]

37. Vemuri, K.P.; Bandaru, P.R. Geometrical considerations in the control and manipulation of conductive heat flux in multilayered thermal metamaterials. Appl. Phys. Lett. 2013, 103, 133111. [CrossRef]

38. Yang, T.; Vemuri, K.P.; Bandaru, P.R. Experimental evidence for the bending of heat flux in a thermal metamaterial. Appl. Phys. Lett. 2014, 105, 83908. [CrossRef]

39. Canbazoglu, F.M.; Vemuri, K.P.; Bandaru, P.R. Estimating interfacial thermal conductivity in metamaterials through heat flux mapping. Appl. Phys. Lett. 2015, 106, 143904. [CrossRef]

40. Xu, G.Q.; Zhang, H.C.; Jin, Y.; Li, S.; Li, Y. Control and design heat flux bending in thermal devices with transformation optics. Opt. Express 2017, 25, A419-A431. [CrossRef] [PubMed]

41. Vemuri, K.P.; Canbazoglu, F.M.; Bandaru, P.R. Guiding conductive heat flux through thermal metamaterials. Appl. Phys. Lett. 2014, 105, 193904. [CrossRef]

42. Park, G.; Kang, S.; Lee, H.; Choi, W. Tunable multifunctional thermal metamaterials: Manipulation of local heat flux via assembly of unit-cell thermal shifters. Sci. Rep. 2017, 7, 41000. [CrossRef] [PubMed] 
43. Peralta, I.; Fachinotti, V.D. Optimization-based design of heat flux manipulation devices with emphasis on fabricability. Sci. Rep. 2017, 7, 6261. [CrossRef] [PubMed]

44. Torabi, M.; Zhang, K. Heat transfer and thermodynamic performance of convective-radiative cooling double layer walls with temperature-dependent thermal conductivity and internal heat generation. Energy Convers. Manag. 2015, 89, 12-23. [CrossRef]

45. Zhang, H.C.; Xu, G.Q.; Yu, H.Y.; Li, Y.; Wei, Y.Q. Investigating entropy generation in a thermal cloak corresponding different material layer number. ASME J. Heat Trans. 2017, 139, 54501. [CrossRef] 